\title{
Field-induced quantum breakdown of superconductivity in magnesium diboride
}

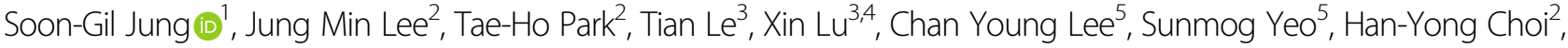 \\ Won Nam Kang ${ }^{2}$ and Tuson Park $\mathbb{D}^{1}$
}

\begin{abstract}
The quantum breakdown of superconductivity (QBS) is the reverse, comprehensive approach to the appearance of superconductivity. A quantum phase transition from superconducting to insulating states tuned by using nonthermal parameters is of fundamental importance to understanding the superconducting (SC) phase but also to practical applications of SC materials. However, the mechanism of the transition to a nonzero resistive state deep in the SC state is still under debate. Here, we report a systematic study of $\mathrm{MgB}_{2}$ bilayers with different thickness ratios for undamaged and damaged layers fabricated by low-energy iron-ion irradiation. The field-induced QBS is discovered at a critical field of 3.2 Tesla $\left(=H_{c}\right)$, where the quantum percolation model best explains the scaling of the magnetoresistance near $H_{c}$. As the thickness of the undamaged layer is increased, strikingly, superconductivity is recovered from the insulating state associated with the QBS, showing that destruction of quantum phase coherence among Cooper electron pairs is the origin of the QBS.
\end{abstract}

\section{Introduction}

Disorder in materials is undesirable because it prevents investigations of the intrinsic properties of the material. In correlated superconductors, however, disorder can be useful in manipulating superconducting properties. Even though the superconducting (SC) transition temperature $\left(T_{\mathrm{c}}\right)$ is decreased with a moderate level of disorder, the current-carrying capacity in high magnetic fields can be improved because disordered regions prevent vortex creep $^{1,2}$. Strong disorder, however, breaks the coherence of SC electron pairs and gives rise to a change from the $\mathrm{SC}$ ground state to an insulating or non-SC metallic state at the quantum breakdown of superconductivity $(\mathrm{QBS})^{3-14}$. As disorder increases, superconducting islands may appear in the destroyed background of non-SC regions owing to inhomogeneous suppression of superconductivity $^{3-6,11}$. Because the presence of SC islands and

\footnotetext{
Correspondence: Tuson Park (tp8701@skku.edu)

${ }^{1}$ Center for Quantum Materials and Superconductivity (CQMS), Department of Physics, Sungkyunkwan University, Suwon 16419, South Korea

${ }^{2}$ Department of Physics, Sungkyunkwan University, Suwon 16419, South Korea

Full list of author information is available at the end of the article
}

the phase coherence among them are important to the emergence of superconductivity, numerous experimental and theoretical efforts have been expended to understand the role of disorder in the SC state ${ }^{3-14}$. However, systematic control of disorder in correlated superconductors has been technically difficult.

Ion beam irradiation is one approach that provides engineered disorder in SC materials. For example, lowenergy ion irradiation produces atomic lattice displacements in crystalline materials because elastic scattering between incident ions and atoms in the target material is dominant ${ }^{15-17}$. In addition, the tunability and simplicity of this technique make the localization of Cooper pairs in disordered SC systems easier to control. The quasi-twodimensional superconductor $\mathrm{MgB}_{2}$ is an ideal example for this study because of its high $T_{\mathrm{c}}$ of $40 \mathrm{~K}$ and the possibility of localizing Cooper pairs near the QBS in disordered $\mathrm{MgB}_{2}{ }^{18,19}$.

In this work, we report magnetic-field-induced quantum breakdown of superconductivity in $\mathrm{MgB}_{2}$ thin films via irradiation with $140-\mathrm{keV}$ Fe-ion beams. The metallic characteristics of $\mathrm{MgB}_{2}$ were significantly suppressed after 
low-energy ion irradiation. When subjected to a magnetic field, the irradiated $\mathrm{MgB}_{2}$ films revealed QBS at a critical field $H_{\mathrm{c}}$ of 3.2 Tesla. The superconductor-to-insulator transition (SIT) within the SC state indicates that SC islands are formed in the $\mathrm{MgB}_{2}$ film due to irradiation and that phase coherence between them is destroyed by the applied magnetic field. When the irradiated $\mathrm{MgB}_{2}$ layer $\left(S_{\mathrm{D}}\right)$ is in contact with a pristine, undamaged $\mathrm{MgB}_{2}$ layer $\left(S_{\mathrm{S}}\right)$, the SIT-like behavior disappears. Here, the two layers comprise a $S_{\mathrm{D}} / S_{\mathrm{S}}$ bilayer. Suppressed superconductivity in the damaged $S_{\mathrm{D}}$ layer was gradually restored to that of its pristine state as the thickness of the $S_{\mathrm{S}}$ was increased, revealing a giant superconducting proximity effect (GSPE). These discoveries suggest that SC islands embedded in the normal matrix are the origin of GSPE and QBS in magnesium diboride bilayers and should provide valuable insights into the development of SC junctions and their applications.

\section{Materials and methods}

$\mathrm{MgB}_{2}$ thin films were fabricated using a hybrid physicalchemical vapor deposition (HPCVD) method, which is an effective technique for fabricating high-quality $\mathrm{MgB}_{2}$ thin films ${ }^{20,21}$. For Fe-ion irradiation, $c$-axis-oriented $\mathrm{MgB}_{2}$ thin films with various total thicknesses $(t)$ of 215 (MB215nm), 440 (MB440nm), and $600 \mathrm{~nm}$ (MB600nm) were fabricated on $c$-cut $\mathrm{Al}_{2} \mathrm{O}_{3}$ substrates at a growth temperature of $680^{\circ} \mathrm{C}$, a pressure of 100 Torr and flow rates for $\mathrm{H}_{2}$ and $\mathrm{B}_{2} \mathrm{H}_{6}$ of 100 and $50 \mathrm{sccm}$, respectively. The fabrication and quality of the films were described in detail in previous studies ${ }^{20,22}$.

Fe ions with an energy of $140 \mathrm{keV}$ were used to irradiate $\mathrm{MgB}_{2}$ thin films at the Korea Multi-purpose Accelerator Complex (KOMAC) at room temperature. Samples MB215nm, MB440nm, and MB600nm were placed together in a sample holder for irradiation. The mean projected ion range $\left(R_{\mathrm{p}}\right)$ and the thickness $t_{\mathrm{D}}$ of the irradiated $\mathrm{MgB}_{2}$ thin films were simulated with Monte Carlo simulations. The Stopping and Range of Ions in Matter (SRIM) (The projected range of Fe ions and the damage events in the $\mathrm{MgB}_{2}$ thin film were calculated using the SRIM software (http://www.srim.org/).), a $\mathrm{MgB}_{2}$ target density of $2.57 \mathrm{~g} / \mathrm{cm}^{3}$ and averaged displacement threshold energy values of $20 \mathrm{eV}(\mathrm{Mg})$ and $46 \mathrm{eV}(\mathrm{B})$ were used $^{23}$. As the total damage and the damage profile depend on dose level for the same incident energy, different dose levels were used to obtain various $t_{\mathrm{D}}$ values ranging from 126 to $203 \mathrm{~nm}$ (see Figs. S1 and S2 in SI).

Changes in the $c$-axis lattice constants of $\mathrm{MgB}_{2}$ layers damaged by $\mathrm{Fe}$-ion irradiation were investigated by $\mathrm{X}$-ray diffraction $(\theta-2 \theta$ scan $)$ before and after irradiation. Values of $T_{\mathrm{c}}$ for the $\mathrm{MgB}_{2}$ bilayers before and after irradiation were determined by using the temperature dependence of electrical resistivity $(\rho)$, as obtained using a Physical
Property Measurement System (PPMS 9T, Quantum Design). Bulk superconductivity was evaluated by using the zero-field-cooled (ZFC) dc magnetization (M) obtained with a Magnetic Property Measurement System (MPMS $5 \mathrm{~T}$, Quantum Design). A standard four-probe method was used for electrical-resistivity measurements. Measurements of the magnetic field dependence of resistivities of the MB215nm bilayers with $\gamma_{\mathrm{t}}=0.12$ and 0.06 were performed using the PPMS. The temperature dependences of the zero-bias conductances (ZBCs) of pristine $M B 600 \mathrm{~nm}$ and the bilayer with $\gamma_{\mathrm{t}}=1.96$ were measured in various magnetic fields by soft point-contact spectroscopy (SPCS) in the PPMS (14 T, Quantum Design). Soft-point contacts on the surface of MB600nm were made with a gold wire (diameter: $30 \mu \mathrm{m}$ ) imbedded in a drop of $\mathrm{Ag}$ paint at the end $\mathrm{nm}$. The total contact diameter of the $\mathrm{Ag}$ paint on the film surface was $\sim 50-100 \mu \mathrm{m}$, where thousands of parallel nanoscale junctions were assumed to exist between individual $\mathrm{Ag}$ particles and the film surface for SPCS ${ }^{24}$.

\section{Results and discussion}

Figure 1a schematically illustrates the effects on a pristine crystal caused by low-energy iron-ion irradiation. Because elastic scattering of the incident ions by nuclei in the materials is dominant for low-energy ion irradiation, lattice displacements, together with the formation of vacancies and interstitials, take place in the irradiated crystal, leading to changes in SC critical properties ${ }^{15-17}$. To probe tunable SC properties by introducing lattice disorder, we used low-energy iron-ion irradiation to fabricate $\mathrm{MgB}_{2}$ bilayers composed of $S_{\mathrm{D}}$ (damaged $\mathrm{MgB}_{2}$ layer) and $S_{\mathrm{S}}$ (undamaged $\mathrm{MgB}_{2}$ layer), as depicted in Fig. 1b. The superconductivity of $S_{\mathrm{D}}$ was destroyed by the disorder produced from ion irradiation, whereas the superconductivity of $S_{\mathrm{S}}$ was maintained as that of the pristine state because it was unaffected by irradiation.

Figure 1c shows a representative enlarged view near the (002) peaks of X-ray diffraction (XRD) patterns for $\mathrm{MgB}_{2}$ with a thickness of $600 \mathrm{~nm}$ (see Fig. S3 in SI). The (002) peak splitting indicates the separation of films into two layers after ion irradiation, and the peak position for the irradiated part of the film shifted to a smaller angle as the dose of irradiating $\mathrm{Fe}$ ions was increased; this corresponded to an increase in the $c$-axis lattice constant, as presented in Fig. 1d. The appearance of a secondary peak at a lower angle reflects the formation of $\mathrm{MgB}_{2}$ bilayers owing to the separation of damaged and undamaged $\mathrm{MgB}_{2}$ layers. All $\mathrm{MgB}_{2}$ films with thicknesses of 215, 440, and $600 \mathrm{~nm}$, which are identified as MB215nm, MB440nm, and MB600nm, respectively, show the same dose dependences of the $c$-axis lattice constant and changes in the $c$-axis lattice constant $\Delta c$, which are plotted as the left and right ordinates of Fig. 1d, respectively. The 
a

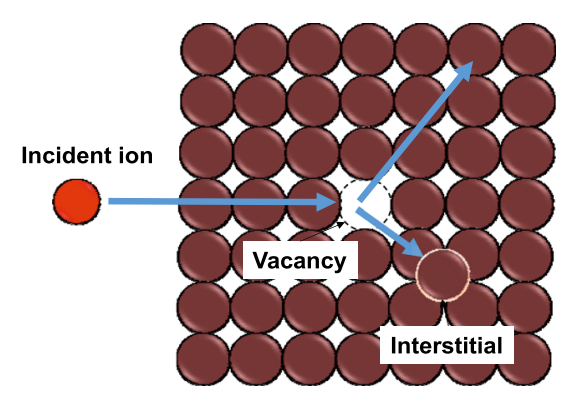

C



b



d

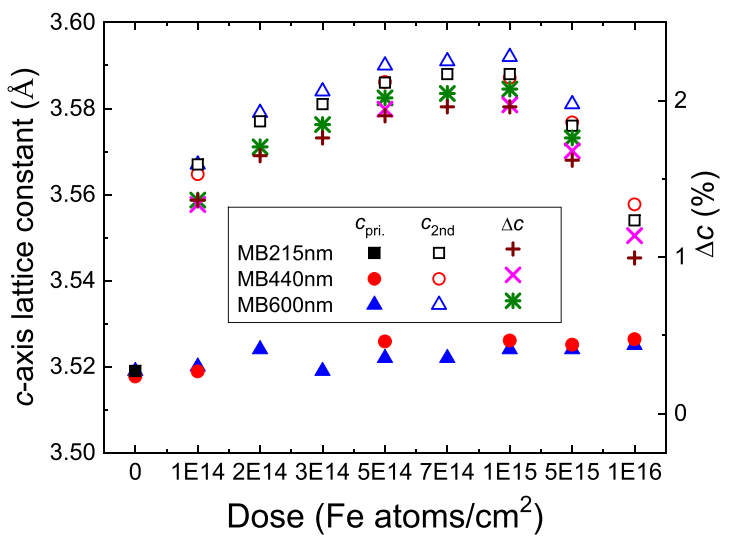

Fig. 1 Formation of $\mathbf{M g B}_{2}$ bilayers by $\mathbf{1 4 0 - k e V ~ F e - i o n ~ i r r a d i a t i o n . ~ S c h e m a t i c s ~ o f ~ a ~ d i s p l a c e m e n t ~ d a m a g e ~ c a u s e d ~ b y ~ e l a s t i c ~ s c a t t e r i n g ~ b e t w e e n ~}$ the incident Fe ions and $\mathrm{Mg} / \mathrm{B}$ atoms and $\mathbf{b} \mathrm{MgB}_{2}$ bilayer $\left(S_{D} / S_{S}\right)$ formed by ion irradiation. The $S_{D}$ layer, with a thickness of $t_{D}$, is the $M g B_{2}$ layer damaged by ion irradiation, whereas the $S_{S}$ layer, with a thickness of $t_{S}$, is the undamaged $M B_{2}$ layer. $\mathbf{c}$ Enlarged views near the (002) peaks of XRD patterns for Fe-ion-irradiated MB600nm. The (002) peak of MB600nm is split into two, with the first $\left(1^{\text {st }}\right)$ and the second $\left(2^{\text {nd }}\right)$ peaks reflecting the formation of $\mathrm{MgB}_{2}$ bilayers. The $1^{\text {st }}$ and $2^{\text {nd }}$ peaks correspond to the undamaged and damaged $\mathrm{MgB}_{2}$ layers, respectively, as indicated by the arrows. d The $c$-axis lattice parameters of the pristine film and the bilayer, where $c_{\text {pri. }}$ from the $1^{\text {st }}$ peak and $c_{2 \text { nd }}$ from the $2^{\text {nd }}$ peak are similar for all samples irrespective of the thicknesses of the films and the irradiation dose levels.

$\Delta c$ values calculated from the position of the second $\left(2^{\text {nd }}\right)$ peak as a function of the dose are similar for all samples regardless of the thicknesses of the films, showing that the damaged $\mathrm{MgB}_{2}$ layers in all the films have similar degrees of disorder produced by irradiation.

Figure $2 \mathrm{a}-\mathrm{d}$ contains plots of the electrical resistivities $(\rho)$ of $\mathrm{MgB}_{2}$ films with a total thickness of $215 \mathrm{~nm}$ as a function of temperature for the pristine layer and the bilayers $\left(S_{\mathrm{D}} / S_{\mathrm{S}}\right)$ with $\gamma_{\mathrm{t}}=0.54,0.30$, and 0.06 , respectively (see Fig. S4 in SI). Here, $\gamma_{\mathrm{t}}$ is the ratio $\left(\gamma_{\mathrm{t}}=t_{\mathrm{S}} / t_{\mathrm{D}}\right)$ between the thickness $t_{\mathrm{S}}$ of $S_{\mathrm{S}}$ and thickness $t_{\mathrm{D}}$ of $S_{\mathrm{D}}$ layers, and the value of $\rho(T)$ is normalized by the resistivity value $\left(\rho_{\mathrm{n}}\right)$ at the onset temperature of the SC transition for each sample. The $\rho(T)$ for pristine MB215nm decreased with decreasing temperature, exhibiting metallic behavior. As the thickness ratio $\gamma_{\mathrm{t}}$ decreased, the metallic characteristic was suppressed owing to an increase in the relative thickness of disordered layer $S_{\mathrm{D}}$. For the bilayer with $\gamma_{\mathrm{t}}=$ $0.06, \rho(T)$ reached a minimum near $112 \mathrm{~K}$ and increased with further decreases in temperature, exhibiting insulating behavior. Below $40 \mathrm{~K}, \rho(T)$ began to decrease because of the SC islands formed in the irradiated films.

The dependence on the thickness ratio $\gamma_{t}$ of the SC volume fraction of the $\mathrm{MgB}_{2}$ bilayers is presented in Fig. 2e-h, which shows the zero-field-cooled (ZFC) and field-cooled (FC) dc magnetizations measured at 5 Oe for all samples (see Fig. S5 in SI). The SC volume fraction of pristine MB215nm at $2 \mathrm{~K}$ was assumed to be $100 \%$, while that of bilayers with different values of $\gamma_{\mathrm{t}}$ was estimated relative to pristine $M B 215 \mathrm{~nm}$. As $\gamma_{\mathrm{t}}$ decreased, the $\mathrm{SC}$ volume fraction decreased, and the $\mathrm{SC}$ transition width of the ZFC $M(T)$ curve broadened because the magnetic field easily penetrated into disordered $\mathrm{MgB}_{2} \mathrm{SC}$ regions. When the value of the thickness ratio $\gamma_{\mathrm{t}}$ was 0.06 , interestingly, the ZFC and the FC $M(T)$ for the bilayer showed clear separation at quite high temperatures even though the SC volume fraction was $<0.1 \%$. Taken together with $\rho(T)$, the small volume fraction of $M(T)$ resulting in the film with $\gamma_{t}=0.06$ is suggestive of the formation of local $\mathrm{SC}$ regions in the damaged $\mathrm{MgB}_{2}$ layer. 




$\mathbf{e}$





$f$

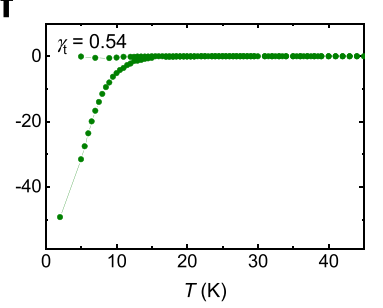

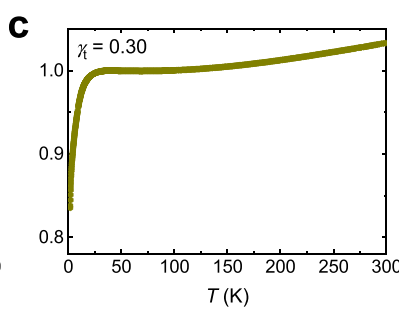

g

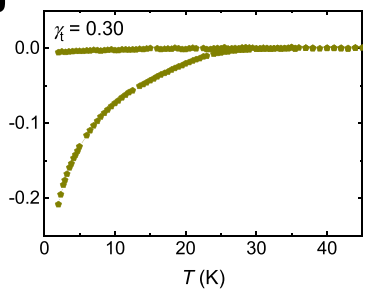

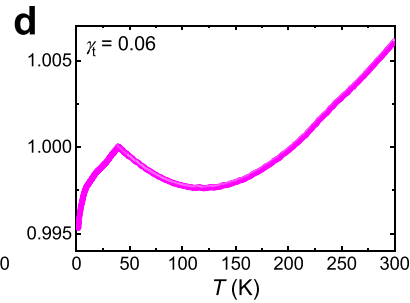

h

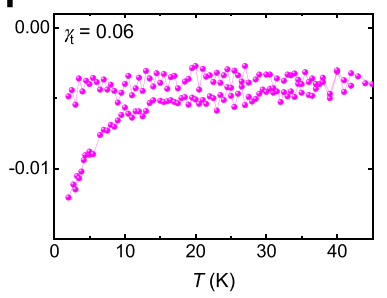

Fig. 2 Temperature dependences of the electrical resistivity and the magnetizations of bilayers of $\mathbf{M B 2 1 5 n m}$. a- $\mathbf{d}$ Representative temperature dependences of electrical resistivity $(\rho)$ are shown for pristine $M B 215 \mathrm{~nm}$ and $\mathrm{MgB}_{2}$ bilayers with $\gamma_{\mathrm{t}}=0.54,0.30$, and 0.06 . Here, $\rho(T)$ is normalized to its value at $T_{c}$ onset $\left(=\rho_{n}\right)$ for each sample for comparison. $\mathbf{e}-\mathbf{h}$ Temperature dependences of zero-field-cooled (ZFC) and field-cooled (FC) dc magnetizations (M) for the same sample set as in Fig. 2a-d; the ZFC and FC $M$ data were measured at 5 Oe for all samples. The SC volume fraction inferred from the ZFC M of the pristine film was assumed to be $100 \%$ at $2 \mathrm{~K}$, while those of the bilayers with different values of $\gamma_{\mathrm{t}}$ were estimated in comparison with the ZFC M value of the pristine film.

Superconductivity in disordered SC systems is not suppressed homogeneously, but disorder-induced inhomogeneity does occur, and local SC regions can be formed. The magnetic field, one of the nonthermal control parameters that can introduce a quantum phase transition at zero Kelvin, is expected to be effective in suppressing phase coherence between local SC regions, i.e., SC islands, thus driving quantum breakdown of superconductivity in disordered SC thin films ${ }^{3-6,11-14,25-28}$. Figure 3a, b presents $\rho(T)$ of $\mathrm{MgB}_{2}$ bilayers with a total thickness $\left(t=t_{\mathrm{D}}+t_{\mathrm{S}}\right)$ of $215 \mathrm{~nm}$ for $\gamma_{\mathrm{t}}=0.12$ and 0.06 , respectively, with several magnetic fields applied perpendicular to the film plane. Evidence for a magnetic-fieldinduced QBS was observed for both bilayers, wherein $\rho(T)$ increased with decreasing temperature below the SC transition temperature, $T_{\mathrm{c}}$. At 0 Tesla, the resistivity gradually decreased with decreasing temperature owing to the superconducting transition in SC islands and the weak correlation between SC islands for $T<T_{\mathrm{c}}$. As the applied magnetic field was increased, the rate of decrease in $\rho(T)$ became weaker because of the suppression of phase coherence between SC islands. At $3 \mathrm{~T}, \rho(T)$ was almost constant, showing a plateau behavior. With a further increase in the field, $\rho(T)$ increased with decreasing temperature, reflecting insulating behavior due to the destruction of interisland coupling (see Fig. S6 in SI). The magnetic field dependences of the resistivities are plotted for various temperatures in the insets of Fig. 3c, d. A crossover from an SC state to an insulating-like state is observed at the critical field $\left(H_{\mathrm{c}}\right)$ near $3.2 \mathrm{~T}$ for both bilayers, indicating that the $\mathrm{SC}$ islands were weakly coupled for $0 \leq H \leq H_{\mathrm{c}}$ but electrically isolated for $H_{\mathrm{c}}<$ $H \leq H_{\mathrm{c} 2}$.

The main panels of Fig. 3c, d reveal scaling of the resistivity as a function of $\left|H-H_{\mathrm{c}}\right| / T^{1 / z v}$ on a semilogarithmic scale. The dynamic exponent $z$ is determined by a characteristic energy $\Omega \propto \xi^{-z}$, where the SC correlation length $\xi(H) \propto\left|H-H_{\mathrm{c}}\right|^{-v}$. The best scaling was observed when the exponent product $z v$ was $7 / 3$, which is consistent with quantum percolation, indicating that quantum breakdown of superconductivity at $H_{\mathrm{c}}$ occurred owing to the destruction of phase coherence between SC islands in disordered $\mathrm{MgB}_{2}$ films ${ }^{3,12,13,29}$ (see Fig. S7 for classical percolation results in SI). We note that the critical resistance at the crossing point of magnetoresistance $H_{\mathrm{c}}$ was considerably smaller than the predicted quantum resistance $\left(R_{\mathrm{Q}}=h / 4 e^{2}=6.45 \mathrm{k} \Omega\right)$ at the quantum critical point $^{3,5,29,30}$, indicating the possibility of an anomalous metallic phase in disordered $\mathrm{MgB}_{2}$ thin films $\mathrm{s}^{3,10,30-35}$. A large charge carrier density and fermionic (unpaired electrons) excitations have been proposed for the origin of unusual metallic behavior and small critical sheet resistance ${ }^{33-35}$. The fact that $\mathrm{MgB}_{2}$ has a relatively large charge carrier density ${ }^{36}$ indicates that the anomalous metallic phase in disordered $\mathrm{MgB}_{2}$ thin films could be associated with contributions from a large number of unpaired electrons to the background conduction bath. However, further studies are required to understand the QBS in quasi-2D $\mathrm{MgB}_{2}$.

Figure 4a shows the $\gamma_{\mathrm{t}}$ dependence of the $\mathrm{SC}$ transition temperature $\left(T_{\mathrm{c}, \mathrm{BL}}\right)$ of $\mathrm{MgB}_{2}$ bilayers, where $T_{\mathrm{c}, \mathrm{BL}}$ was normalized by the $T_{\mathrm{c}}$ of the corresponding pristine $\mathrm{MgB}_{2}$ 

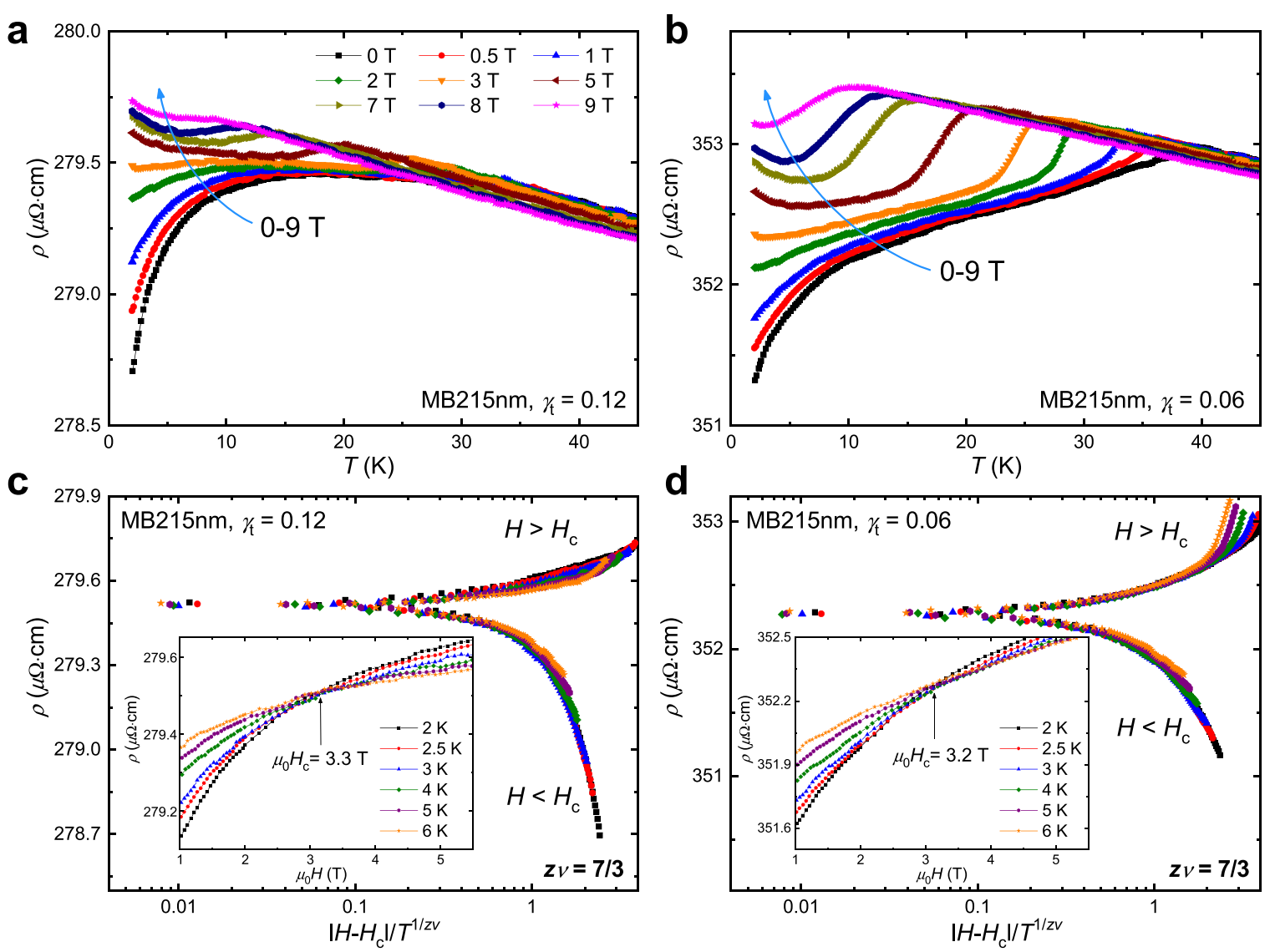

Fig. 3 Quantum breakdown of superconductivity in magnesium diboride. Temperature dependences of the electrical resistivity $(\rho)$ of the MB215nm bilayers with $\gamma_{\mathrm{t}}=(\mathbf{a}) 0.12$ and (b) 0.06 in magnetic fields applied perpendicular to the $a b$ plane. The $\rho(T) \mathrm{s}$ of both MB215nm bilayers are lower at temperatures below $T_{c}$ but show an upturn in the SC state when the magnetic field is larger than $3 \mathrm{~T}$. Magnetoresistivities $\rho(H, T)$ are plotted as functions of $\left|H-H_{c}\right| / T^{1 / 2 v}$ for the bilayers with $\gamma_{t}=(\mathbf{c}) 0.12$ and $(\mathbf{d}) 0.06$. The insets of $(\mathbf{c})$ and $(\mathbf{d})$ show $\rho(H)$ for several temperatures ranging from 2 to $6 \mathrm{~K}$, in which the field dependences of the resistivities for the bilayers with $\gamma_{\mathrm{t}}=0.12$ and 0.06 exhibit single crossing points at 3.3 and $3.2 \mathrm{~T}$, respectively. The magnetoresistivities $\rho(H, T)$ of both bilayers are well scaled with the exponent $z V=7 / 3$, indicating that quantum percolation between SC islands leads to a quantum breakdown of superconductivity (QBS) in the disordered $\mathrm{MgB}_{2}$ layers.

thin film $\left(T_{\mathrm{c}, \text { pri. }}\right)$ (see Figs. S8 and S9 in SI). The $T_{\text {c,pri. }}$ values of MB215nm, MB440nm, and MB600nm were 39.3, 39.7, and $40 \mathrm{~K}$, respectively. As $t_{\mathrm{S}}$ increased, $T_{\mathrm{c}, \mathrm{BL}}$ initially increased rapidly and saturated to $T_{\mathrm{c}, \mathrm{pri}}$. even though the thickness of the damaged layer, $t_{\mathrm{D}}$, was considerably larger than the coherence length of $\mathrm{MgB}_{2}$ $\left(\xi_{\mathrm{MgB} 2} \sim 7 \mathrm{~nm}\right)$. The presence of proximity effects up to the surface of $S_{\mathrm{D}}$ was evidenced by SPCS (see Figs. S10 and S11 in SI). Although the behavior of $T_{\mathrm{c}, \mathrm{BL}}$ with respect to the thickness ratio $\gamma_{\mathrm{t}}$ was similar to results for the proximity effect in $N / S$ bilayers, the length scale of the proximity effect in $S_{\mathrm{D}} / S_{\mathrm{S}}$ was considerably larger than the value predicted using conventional theory ${ }^{37,38}$. Here, the red solid line in Fig. 4a was obtained from the Werthamer theory in which the spatial variation in the BCS electron-electron interaction is considered ${ }^{37}$.

The long-range proximity effect, the so-called giant superconducting proximity effect (GSPE), with an anomalously large leakage distance for Cooper pairs was often observed between two superconductors composed of the same materials but with different $T_{\mathrm{c}}$ values $^{39-44}$. For example, when an optimally doped $\mathrm{La}_{1.85} \mathrm{Sr}_{0.15} \mathrm{CuO}_{4}$ (LSCO) layer with $T_{\mathrm{c}} \approx 45 \mathrm{~K}$ was in contact with an underdoped $\mathrm{La}_{2} \mathrm{CuO}_{4-d}$ (LCO) layer with $T_{\mathrm{c}} \approx 25 \mathrm{~K}$, GSPE was observed at temperatures higher than the $T_{\mathrm{c}}$ of $\mathrm{LCO}^{41}$. Several scenarios, such as phase fluctuations, amplitude fluctuations, and proximity-induced interface superconductivity, were proposed to explain the origin of the GSPE ${ }^{43,45-48}$. Figure $4 \mathrm{~b}$ is a simple cartoon used to describe the GSPE in $\mathrm{MgB}_{2}$ bilayers $\left(S_{\mathrm{D}} / S_{\mathrm{S}}\right)$, and the unusual proximity length scale may be understood by the presence of spatially distributed SC islands in $S_{\mathrm{D}}$. Phase coherence between the SC islands in $S_{\mathrm{D}}$ can be enhanced by leaking of Cooper pairs from $S_{\mathrm{S}}$, sequentially forming strong Josephsonjunction chains between the SC islands. The enhanced length scale from the proximity effect, in turn, gives rise to the suppression of the QBS in $S_{\mathrm{D}}^{25,26,46}$. These findings underscore 



Fig. 4 Giant superconducting proximity effect in the $\mathbf{M g B}_{\mathbf{2}}$ bilayer. a The $\mathrm{SC}$ transition temperature $\left(T_{c, B L}\right)$ of $\mathrm{MgB}_{2}$ bilayers as a function of $\gamma_{\mathrm{t}}$ where $T_{c, B L}$ is normalized to the $T_{c}$ of the corresponding pristine $\mathrm{MgB}_{2}$ thin film $\left(T_{c, \text { pri }}\right)$. Square, circular, and triangular symbols represent the three films with different thicknesses of $215 \mathrm{~nm}$ (MB215nm), $440 \mathrm{~nm}$ (MB440nm), and $600 \mathrm{~nm}$ (MB600nm), respectively. The thickness $t_{D}$ of layer $S_{D}$ $(126-203 \mathrm{~nm})$ was controlled by varying the dose of irradiated Fe ions. The red solid line is a fitting curve obtained by using the Werthamer theory that considers the spatial variation in the attractive electron-electron interaction. $\mathbf{b}$ Schematic view of the GSPE in the bilayer $S_{D} / S_{S}$. SC islands (SCI) in the $S_{D}$ layer sequentially form Josephson-junction chains between the SC islands. Phase coherence between the SC islands is enhanced by Cooper pairs that leak from the $S_{S}$ layer, which can lead to a power-law decay of the order parameter as a function of the distance from the $S_{D} / S_{S}$ boundary, $\Delta(x) \propto 1 / x$, rather than an exponential decay of $\Delta(x) \propto \exp \left(-x / \xi_{D}\right)$. Here, $\xi_{D}$ is the leaking distance of the order parameter to the $S_{D}$ layer from the boundary of the $S_{D}$ and $S_{S}$ layers. The regular array of SC islands is exaggerated for simplicity, and the spacing between them could be larger than the coherence length of $\mathrm{MgB}_{2}$.

that $\mathrm{SC}$ islands formed in the normal matrix are the origin of the GSPE in the $\mathrm{MgB}_{2}$ bilayers composed of a damaged layer $\left(S_{\mathrm{D}}\right)$ and an undamaged layer $\left(S_{\mathrm{S}}\right)$.

\section{Conclusion}

In conclusion, we observe field-induced quantum breakdown of superconductivity in disordered $\mathrm{MgB}_{2}$ and a local pairing-induced GSPE in $S_{\mathrm{D}} / S_{\mathrm{S}} \mathrm{MgB}_{2}$ bilayers 
fabricated with low-energy ion irradiation. When an applied magnetic field is sufficiently high to break phase coherence among SC islands in $S_{\mathrm{D}}$, QBS is observed at a critical field $H_{\mathrm{c}}$, the scaling of which is consistent with that of the quantum percolation model. As the thickness of $S_{\mathrm{S}}$ is increased, the suppressed superconductivity of $S_{\mathrm{D}}$ is recovered to that of the pristine state, and the QBS does not occur, not even at fields larger than $H_{\mathrm{c}}$. Taken together, these findings underpin the conclusion that local superconducting pairing is the origin of $\mathrm{QBS}$ and the GSPE in the $\mathrm{MgB}_{2}$ bilayer.

\section{Acknowledgements}

We thank J. D. Thompson for helpful discussions. We wish to acknowledge the support of the accelerator group and operators of KOMAC (KAERI). This study was supported by the National Research Foundation (NRF) of Korea through a grant funded by the Korean Ministry of Science and ICT (No.

2012R1A3A2048816 and 2021R1A2C2010925) and by the Basic Science Research Program through the NRF of Korea funded by the Ministry of Education (NRF-2018R1D1A1B07048987, NRF-2019R1F1A1055284, and NRF2020R1I1A1A01054852). X.L. acknowledges support from the National Key Research \& Development Program of China (Grants No. 2016FYA0300402 and No. 2017YFA0303101) and the National Natural Science Foundation of China (Grants No. 11674279).

\section{Author details}

${ }^{1}$ Center for Quantum Materials and Superconductivity (CQMS), Department of Physics, Sungkyunkwan University, Suwon 16419, South Korea. ${ }^{2}$ Department of Physics, Sungkyunkwan University, Suwon 16419, South Korea. ${ }^{3}$ Center for Correlated Matter and Department of Physics, Zhejiang University, Hangzhou 310058, People's Republic of China. ${ }^{4}$ Collaborative Innovation Center of Advanced Microstructures, Nanjing University, Nanjing 210093, People's Republic of China. ${ }^{5}$ Korea Multi-purpose Accelerator Complex, Korea Atomic Energy Research Institute, Gyeongju, Gyeongbuk 38180, Republic of Korea

\section{Competing interests}

The authors declare no competing interests.

\section{Publisher's note}

Springer Nature remains neutral with regard to jurisdictional claims in published maps and institutional affiliations.

Supplementary information The online version contains supplementary material available at https://doi.org/10.1038/s41427-021-00323-x.

Received: 24 February 2021 Revised: 9 June 2021 Accepted: 15 June 2021. Published online: 23 July 2021

\section{References}

1. Bugoslavsky, Y. et al. Enhancement of the high-magnetic-field critical current density of superconducting $\mathrm{MgB}_{2}$ by proton irradiation. Nature $\mathbf{4 1 1}, 561-563$ (2001).

2. Fang, L. et al. Huge critical current density and tailored superconducting anisotropy in $\mathrm{SmFeAsO}_{0.8} \mathrm{~F}_{0.15}$ by low-density columnar-defect incorporation. Nat. Commun. 4, 2655 (2013).

3. Sacépé, B., Feigel'man, M. \& Klapwijk, T. M. Quantum breakdown of superconductivity in low-dimensional materials. Nat. Phys. 16, 734-746 (2020).

4. Sacépé, B. et al. Localization of preformed Cooper pairs in disordered superconductors. Nat. Phys. 7, 239-244 (2011).

5. Gantmakher, V. F. \& Dolgopolov, V. T. Superconductor-insulator quantum phase transition. Phys. Usp. 53, 1-49 (2010).

6. Dubi, Y., Meir, Y. \& Avishai, Y. Nature of the superconductor-insulator transition in disordered superconductors. Nature 449, 876-880 (2007).
7. Micnas, R., Ranninger, J. \& Robaszkiewicz, S. Superconductivity in narrow-band systems with local nonretarded attractive interactions. Rev. Mod. Phys. 62, 113-171 (1990).

8. Nozières, P. \& Schmitt-Rink, S. Bose condensation in an attractive fermion gas: from weak to strong coupling superconductivity. J. Low. Temp. Phys. 59, 195-211 (1985)

9. Bouadim, K., Loh, Y. L., Randeria, M. \& Trivedi, N. Single- and two-particle energy gaps across the disorder-driven superconductor-insulator transition. Nat. Phys. 7, 884-889 (2011).

10. Chen, Z. et al. Carrier density and disorder tuned superconductormetal transition in a two-dimensional electron system. Nat. Commun. 9, 4008 (2018)

11. Ghosal, A., Randeria, M. \& Trivedi, N. Inhomogeneous pairing in highly disordered s-wave superconductors. Phys. Rev. B 65, 014501 (2001).

12. Steiner, M. A., Breznay, N. P. \& Kapitulnik, A. Approach to a superconductor-toBose-insulator transition in disordered films. Phys. Rev. B 77, 212501 (2008).

13. Schneider, R., Zaitsev, A. G., Fuchs, D. \& Löhneysen, H. V. Superconductorinsulator quantum phase transition in disordered FeSe thin films. Phys. Rev. Lett. 108, 257003 (2012).

14. Fisher, M. P. A., Weichman, P. B., Grinstein, G. \& Fisher, D. S. Boson localization and the superfluid-insulator transition. Phys. Rev. B 40, 546-570 (1989).

15. Jung, S.-G. et al. Giant proximity effect in single-crystalline $\mathrm{MgB}_{2}$ bilayers. Sci. Rep. 9, 3315 (2019).

16. Jung, S.-G. et al. Influence of carbon-ion irradiation on the superconducting critical properties of $\mathrm{MgB}_{2}$ thin films. Supercond. Sci. Technol. 32, 025006 (2019).

17. Parkin, D. M. Radiation effects in high-temperature superconductors: a brief review. Metall. Trans. A 21A, 1015-1019 (1990).

18. Putti, M., Vaglio, R. \& Rowell, J. M. Radiation effects on $\mathrm{MgB}_{2}$ : a review and a comparison with A15 superconductors. Supercond. Sci. Technol. 21, 043001 (2008).

19. Long, Z., Stewart, M. D. \& Valles, J. M. Super-weakly coupled superconductivity in ultrathin superconductor-normal-metal bilayers. Phys. Rev. B 73, 140507(R) (2006).

20. Seong, W. K., Oh, S. \& Kang, W. N. Perfect domain-lattice matching between $\mathrm{MgB}_{2}$ and $\mathrm{Al}_{2} \mathrm{O}_{3}$ : single-crystal $\mathrm{MgB}_{2}$ thin films grown on sapphire. Jpn. J. Appl. Phys. 51, 083101 (2012).

21. Zeng, $X$. et al. In situ epitaxial $\mathrm{MgB}_{2}$ thin films for superconducting electronics. Nat. Mater. 1, 35-38 (2002).

22. Jung, S.-G., Seong, W. K. \& Kang, W. N. Flux pinning mechanism in singlecrystalline $\mathrm{MgB}_{2}$ thin films. J. Phys. Soc. Jpn. 82, 114712 (2013).

23. Konobeyev, A. Y. U., Fischer, U., Korovin, Y. U. A. \& Simakov, S. P. Evaluation of effective threshold displacement energies and other data required for the calculation of advanced atomic displacement cross-sections. Nucl. Energy Technol. 3, 169-175 (2017).

24. Gonnelli, R. S. \& Daghero, D. N. Probing multiband superconductivity by pointcontact spectroscopy. Supercond. Sci. Technol. 23, 043001 (2010).

25. Escoffier, W., Chapelier, C., Hadacek, N. \& Villégier, J.-C. Anomalous proximity effect in an inhomogeneous disordered superconductor. Phys. Rev. Lett. 93, 217005 (2004).

26. Kresin, V. Z., Ovchinnikov, Y. N. \& Wolf, S. A. Inhomogeneous superconductivity and the "pseudogap" state of novel superconductors. Phys. Rep. 431, 231-259 (2006).

27. Seidler, G. T., Rosenbaum, T. F. \& Veal, B. W. Two-dimensional superconductorinsulator transition in bulk single-crystal $\mathrm{YBa}_{2} \mathrm{Cu}_{3} \mathrm{O}_{6.38}$. Phys. Rev. B 45, 10162-10164 (1992).

28. Zhang, G. et al. Superconductor-insulator transition driven by pressuretuned intergrain coupling in nanodiamond films. Phys. Rev. Mater. 3, 034801 (2019)

29. Lee, D.-H., Wang, Z. \& Kivelson, S. Quantum percolation and plateau transitions in the quantum Hall effects. Phys. Rev. Lett. 70, 4130-4133 (1993).

30. Fisher, M. P. A., Grinstein, G. \& Girvin, S. M. Presence of quantum diffusion in two dimensions: Universal resistance at the superconductor-insulator transition. Phys. Rev. Lett. 64, 587-590 (1990).

31. Yang, $C$. et al. Intermediate bosonic metallic state in the superconductorinsulator transition. Science 366, 1505-1509 (2019).

32. Feigel'man, M. V. \& Larkin, A. I. Quantum superconductor-metal transition in a 2D proximity-coupled array. Chem. Phys. 235, 107-114 (1998).

33. Aubin, $\mathrm{H}$. et al. Magnetic-field-induced quantum superconductor-insulator transition in $\mathrm{Nb}_{0.15} \mathrm{Si}_{0.85}$. Phys. Rev. B 73, 094521 (2006).

34. Zeng, S. et al. Two-dimensional superconductor-insulator quantum phase transitions in an electron-doped cuprate. Phys. Rev. B 92, 020503(R) (2015). 
35. Breznay, N. P., Tendulkar, M., Zhang, L., Lee, S.-C. \& Kapitulnik, A. Superconductor to weak-insulator transitions in disordered tantalum nitride films. Phys. Rev. B 96, 134522 (2017).

36. Kang, W. N. et al. Hole carrier in $\mathrm{MgB}_{2}$ characterized by Hall measurements. Appl. Phys. Lett. 79, 982 (2001).

37. Werthamer, N. R. Theory of the superconducting transition temperature and energy gap function of superposed metal films. Phys. Rev. 132, 2440 (1963).

38. de Gennes, P. G. Boundary effects in superconductors. Rev. Mod. Phys. 36, 225 (1964).

39. Morenzoni, E. et al. The Meissner effect in a strongly underdoped cuprate above its critical temperature. Nat. Commun. 2, 272 (2011).

40. Decca, R. S., Drew, H. D., Osquiguil, E., Maiorov, B. \& Guimple, J. T. Anomalous proximity effect in underdoped $\mathrm{YBa}_{2} \mathrm{Cu}_{3} \mathrm{O}_{6+x}$ Josephson junctions. Phys. Rev. Lett. 85, 3708-3711 (2000).

41. Bozovic, I. et al. Giant proximity effect in cuprate superconductors. Phys. Rev. Lett. 93, 157002 (2004).
42. Kirzhner, T. \& Koren, G. Pairing and the phase diagram of the normal coherence length $\xi_{N}(T, x)$ above $T_{c}$ of $\mathrm{La}_{2-x} \mathrm{Sr}_{x} \mathrm{CuO}_{4}$ thin films probed by the Josephson effect. Sci. Rep. 4, 6244 (2014).

43. Cherkez, $\vee$. et al. Proximity effect between two superconductors spatially resolved by scanning tunneling spectroscopy. Phys. Rev. X 4, 011033 (2014).

44. Kim, J. et al. Visualization of geometric influences on proximity effects in heterogeneous superconductor thin films. Nat. Phys. 8, 464 (2012).

45. Marchand, D., Covaci, L., Berciu, M. \& Franz, M. Giant proximity effect in a phase-fluctuating superconductor. Phys. Rev. Lett. 101, 097004 (2008).

46. Kresin, V., Ovchinnikov, Y. \& Wolf, S. "Giant" Josephson proximity effect. Appl. Phys. Lett. 83, 722-724 (2003).

47. Kresin, V., Ovchinnikov, Y. \& Wolf, S. Inhomogeneous superconductivity and the "pseudogap" state of novel superconductors. Phys. Rep. 431, 231-259 (2006).

48. Covaci, L. \& Marsiglio, F. Proximity effect and Josephson current in clean strong/weak/strong superconducting trilayers. Phys. Rev. B 73, 014503 (2016). 